\title{
Coarse embeddings into a Hilbert space, Haagerup Property and Poincaré inequalities
}

\author{
Romain Tessera*
}

September 10, 2018

\begin{abstract}
We prove that a metric space does not coarsely embed into a Hilbert space if and only if it satisfies a sequence of Poincaré inequalities, which can be formulated in terms of (generalized) expanders. We also give quantitative statements, relative to the compression. In the equivariant context, our result says that a group does not have the Haagerup property if and only if it has relative property $\mathrm{T}$ with respect to a family of probabilities whose supports go to infinity. We give versions of this result both in terms of unitary representations, and in terms of affine isometric actions on Hilbert spaces.
\end{abstract}

\section{Introduction}

\subsection{Obstruction to coarse embeddings}

The notion of expanders has been pointed out by Gromov as an obstruction for a metric space to coarsely embed into a Hilbert space. Recall [JS] (see also [L]) that a sequence of expanders is a sequence of finite connected graphs $\left(X_{n}\right)$ with bounded degree, satisfying the following Poincaré inequality for all $f \in \ell^{2}\left(X_{n}\right)$

$$
\frac{1}{\left|X_{n}\right|^{2}} \sum_{x, y \in X_{n}}|f(x)-f(y)|^{2} \leq \frac{C}{\left|X_{n}\right|} \sum_{x \sim y}|f(x)-f(y)|^{2},
$$

for some constant $C>0$, and whose cardinality $\left|X_{n}\right|$ goes to infinity when $n \rightarrow$ $\infty$. An equivalent formulation in $\ell^{p}[\mathrm{M} 2]$ can be used to prove that expanders do not coarsely embed into $L^{p}$ for any $1 \leq p<\infty$.

\footnotetext{
*The author is supported by the NSF grant DMS-0706486.
} 
It is an open problem whether a metric space with bounded geometry that does not coarsely embed into a Hilbert space admits a coarsely embedded sequence of expanders.

In this paper, we prove that a metric space (not necessarily with bounded geometry) that does not coarsely embed into a Hilbert space admits a coarsely embedded sequence of "generalized expanders". This weaker notion of expanders can be roughly described as a sequence of Poincaré inequalities with respect to finitely supported probability measures on $X \times X$. We also provide similar obstructions for coarse embeddings into families of metric spaces such as $L^{p}$, for every $1 \leq p<\infty$, uniformly convex Banach spaces, and CAT(0) spaces.

For the sake of clarity, we chose to present most of our results first in the case of Hilbert spaces. However, our characterization (see Theorem 13) of the non-existence of coarse embedding into $L^{p}$ deserves some attention. Indeed, our Poincaré inequalities are not equivalent for different values of $2 \leq p<\infty$. This follows from a result of Naor and Mendel [MN] (see also [JR]) saying that $L^{p}$ does not coarsely embed into $L^{q}$ if $2 \leq q<p$. This is different from what happens with real expanders, as having a sequence of expanders prevents from having a coarse embedding into $L^{p}$, for any $1 \leq p<\infty$. In particular, at least without any assumption of bounded geometry, our generalized expanders cannot be replaced by actual expanders.

To conclude, let us remark that finding subspaces of $L^{p}$ for some $p>2$, with bounded geometry, which do not coarsely embed into $L^{2}$, would answer negatively the problem mentioned above.

\subsection{Obstruction to Haagerup Property}

A countable group is said to have the Haagerup property if it admits a proper affine action on a Hilbert space. An obstruction for an infinite countable group to have the Haagerup Property is known as Property T (also called Property FH), which says that every isometric affine action has a fixed point (or equivalently bounded orbits). A weaker obstruction is to have relative property $\mathrm{T}$ with respect to an infinite subset [C1, C2]. The case where this subset is a normal subgroup has been mostly considered, as it has strong consequences. On the other hand, there are examples of groups which do not have relative property $\mathrm{T}$ with respect to any subgroup, but have it with respect to some infinite subset [C1]. The question whether the latter property is equivalent to the negation of Haagerup Property is still open.

In this paper, we partially answer this question by showing that a countable group does not have Haagerup Property if and only if it has relative Property $\mathrm{T}$ 
with respect to a sequence of probabilities whose supports eventually leave every finite subset.

Acknowledgments. I am grateful to Yann Ollivier, Yves de Cornulier, and Bogdan Nica for their useful comments and corrections. I address a special thank to James Lee who pointed to me [M1, Proposition 15.5.2].

\section{Statement of results in the Hilbert case}

In this section, we state our main results concerning embeddings into a Hilbert space. In Section 3, using a slightly more sophisticated vocabulary, we generalize to other geometries.

\subsection{Coarse embeddability into Hilbert spaces and gener- alized expanders}

Let $\mathcal{H}$ denote a separable infinite dimensional Hilbert space. We denote by $|v|$ the norm of a vector in $\mathcal{H}$. Let $X=(X, d)$ be a metric space. For all $r \geq 0$, denote

$$
\Delta_{r}(X)=\left\{(x, y) \in X^{2}, d(x, y) \geq r\right\} .
$$

In this paper, we prove that a metric space that does not coarsely embed into a Hilbert space contains in a weak sense a sequence of expanders. Precisely, following the idea of [T, Section 4.2], let us define

\section{Definition 1.}

- Let $K$ and $r$ be positive numbers. A finite metric space is called a generalized $(K, r)$-expander if there exists a symmetric probability measure $\mu$ supported on $\Delta_{r}(X)$ with the following property. For every map $F: X \rightarrow \mathcal{H}$ satisfying $|F(x)-F(y)| \leq d(x, y)$ for all $(x, y) \in \Delta_{1}(X)$, we have

$$
\operatorname{Var}_{\mu}(F):=\sum_{x, y}|F(x)-F(y)|^{2} \mu(x, y) \leq K^{2} .
$$

- A sequence of finite metric spaces $\left(X_{n}\right)$ is called a sequence of generalized $K$-expander if for every $n \in \mathbf{N}, X_{n}$ is a $\left(K, r_{n}\right)$-expanders, where $r_{n} \rightarrow \infty$.

Recall that a family of metric spaces $\left(X_{i}\right)_{i \in I}$ coarsely embeds into a metric space $Y$ if there exists a family $\left(F_{i}\right)$ of uniformly coarse embeddings of $X_{i}$ into $Y$, i.e. if there are two increasing, unbounded functions $\rho_{-}$and $\rho_{+}$such that

$$
\rho_{-}(d(x, y)) \leq d\left(F_{i}(x), F_{i}(y)\right) \leq \rho_{+}(d(x, y)), \forall x, y \in X_{i}, \forall i \in I .
$$


Proposition 2. A sequence of generalized expanders $\left(X_{n}\right)$ does not coarsely embed into a Hilbert space.

Proof: Let $K>0$ and for all $n \in \mathbf{N}$, let $X_{n}$ is a $\left(K, r_{n}\right)$-expander, with $r_{n} \rightarrow \infty$. For every $n \in \mathbf{N}$, let $F_{n}$ be a map from $X_{n} \rightarrow \mathcal{H}$, and that there exists some increasing function $\rho_{+}$such that $\left|F_{n}(x)-F_{n}(y)\right| \leq \rho_{+}(d(x, y)), \forall x, y \in X_{n}, \forall n \in$ $\mathbf{N}$. As observed in [CTV, Lemmas 2.4 and 3.11], if a metric space (or a family of metric spaces) coarsely embeds into a Hilbert space, we can always assume that the function $\rho_{+}$goes arbitrarily slowly to infinity (this follows from a result of Bochner and Schoenberg [Sch, Theorem 8]). So in particular, we can assume that $\rho_{+}(t) \leq t, \forall t \geq 1$. But then, (2.1) tells us that pairs of points of $X_{n}$, which are at distance $\geq r_{n}$ are sent by $F_{n}$ at distance less than $K$. As $r_{n} \rightarrow \infty$, this implies that any increasing function $\rho_{-}$satisfying

$$
\rho_{-}(d(x, y)) \leq\left|F_{n}(x)-F_{n}(y)\right|, \forall x, y \in X_{n}, \forall n \in \mathbf{N}
$$

would have to be $\leq K$.

Our main result is the following theorem (which is a particular case of Corollary 16).

Theorem 3. A metric space does not coarsely embed into a Hilbert space if and only if it has a coarsely-embedded sequence of generalized expanders.

\subsection{Comparison with the usual notion of expanders}

The usual definition of an expander is a sequence of finite connected graphs $\left(X_{n}\right)$ with degree $\leq k$, satisfying the following Poincaré inequality for all $f \in \ell^{2}\left(X_{n}\right)$

$$
\frac{1}{\left|X_{n}\right|^{2}} \sum_{x, y \in X_{n}}|f(x)-f(y)|^{2} \leq \frac{C}{\left|X_{n}\right|} \sum_{x \sim y}|f(x)-f(y)|^{2},
$$

for some constant $C>0$, and whose cardinality $\left|X_{n}\right|$ goes to infinity when $n \rightarrow$ $\infty$. If $\nu_{n}$ denote the uniform measure on $X_{n} \times X_{n}$, this can be rewritten as

$$
\operatorname{Var}_{\nu_{n}}(f) \leq \frac{C}{\left|X_{n}\right|} \sum_{x \sim y}|f(x)-f(y)|^{2} .
$$

Now, assuming that $f$ is 1-Lipschitz, we have

$$
\operatorname{Var}_{\nu_{n}}(f) \leq k C,
$$

To obtain condition (2.1), we need to replace $\nu_{n}$ by a probability supported far away from the diagonal. To do that, we just notice that at least half of the 
mass of $\nu_{n}$ is actually supported on $\Delta_{r_{n}}$, with $r_{n}=\log _{k}\left(\left|X_{n}\right| / 2\right)$. Indeed, if $r$ is some positive number, the number of pairs of $X_{n} \times X_{n}$ which are at distance $\leq r$ is at most $k^{r}\left|X_{n}\right|$. Hence the proportion of such pairs is $\leq k^{r} /\left|X_{n}\right|$, and the statement follows. Therefore, renormalizing the restriction of $\nu_{n}$ to $\Delta_{r_{n}}$, we obtain a probability $\mu_{n}$ satisfying $\operatorname{Var}_{\mu_{n}}(f) \leq 2 \operatorname{Var}_{\nu_{n}}(f) \leq 2 k C$. Hence, we have proved

Proposition 4. A sequence of expanders satisfying (2.3) with constant $C$, is a sequence of generalized $K$-expanders, with $K=(2 k C)^{1 / 2}$.

\subsection{Haagerup property and relative property $\mathrm{T}$ with re- spect to a family of probabilities}

Recall that a countable group has the Haagerup Property if it acts metrically properly by affine isometries on a Hilbert space. It is well known that this is equivalent to saying that $G$ has a proper Hilbert length (see Section 3 for a definition). On the other hand [C1] a group has relative Property FH with respect to an infinite subset $\Omega$ if every Hilbert length on $G$ is bounded in restriction to $\Omega$.

Definition 5. Let $G$ be a countable group equipped with a proper length function $L_{0}$. Let $\left(\mu_{n}\right)$ be a sequence of probability measures on $G$. We say that $G$ has relative property $F H$ with respect to $\left(\mu_{n}\right)$ there exists $K>0$ such that for every Hilbert length $L$ satisfying

$$
L(g) \leq L_{0}(g), \quad \forall g \in G,
$$

and for every $n \in \mathbf{N}$,

$$
\mathbf{E}_{\mu_{n}}\left(L^{2}\right) \leq K
$$

It is easy to see that this definition does not depend on $L_{0}$.

Note that having relative property $\mathrm{FH}$ with respect to an infinite subset $\Omega=$ $\left\{a_{1}, a_{2}, \ldots\right\}$ corresponds to having relative Property FH with respect to $\left(\mu_{n}\right)$, where $\mu_{n}$ is the Dirac measure at $a_{n}$, for every $n \in \mathbf{N}$.

Theorem 6. A countable group $G$ does not have the Haagerup Property if and only if it has relative Property FH with respect to a sequence of symmetric probability measures $\left(\mu_{n}\right)$, such that for all $n \in \mathbf{N}, \mu_{n}$ is supported on a finite subset of $\left\{g, L_{0}(g) \geq n\right\}$.

Recall that an equivalent formulation of the Haagerup Property (actually the original one) is as follows: there exists a sequence $\left(\phi_{k}\right)$ of positive definite functions on the group such that $\lim _{k \rightarrow \infty} \phi_{k}(g)=1$ for all $g \in G$, and 
$\lim _{g \rightarrow \infty} \phi_{k}(g)=0$ for all $k \in \mathbf{N}$ (in terms of unitary representations, it says that there exists a $C_{0}$ unitary representation with almost-invariant vectors).

An obvious obstruction to the Haagerup Property is [C1] relative property T with respect to an unbounded subset $\Omega$ : every sequence $\left(\phi_{k}\right)$ of positive definite function on $G$ converging to 1 pointwise, converges uniformly in restriction to $\Omega$. In [C1], it is actually proved that relative Property $\mathrm{T}$ with respect to $\Omega$ is equivalent to relative Property FH with respect to $\Omega$. Let us introduce the following definition.

Definition 7. Let $G$ be a countable group. Let $\left(\mu_{n}\right)$ be a sequence of probability measures on $G$. We say that $G$ has relative property $T$ with respect to $\left(\mu_{n}\right)$ if every sequence of positive definite function $\left(\phi_{k}\right)$ that pointwise converges to 1 , satisfies that $\lim _{k \rightarrow \infty} \mathbf{E}_{\mu_{n}}\left(\phi_{k}\right)=1$ uniformly with respect to $n \in \mathbf{N}$.

We have the following theorem

Theorem 8. A countable group $G$ does not have the Haagerup Property if and only if it has relative Property $T$ with respect to a sequence of symmetric probability measures $\left(\mu_{n}\right)$, such that for all $n \in \mathbf{N}, \mu_{n}$ is supported on a finite subset of $\left\{g, L_{0}(g) \geq n\right\}$.

Proof: It is clear that relative Property $\mathrm{T}$ with respect to a sequence of probabilities whose supports go to infinity violates the Haagerup Property. So what we need to prove is the converse, namely, that the negation of Haagerup Property implies relative Property $\mathrm{T}$ with respect to some $\left(\mu_{n}\right)$. By Theorem 6, it is enough to prove that relative Property FH implies relative Property T, which is a straightforward adaptation of the proof of [AW, Theorem 3].

\section{A more general setting, and quantitative state- ments}

In this section, we switch to a slightly different point of view. The statements we want to prove are of the following form: a metric space $X$ that cannot coarsely embed into some class of spaces $\mathcal{M}$ has to satisfy some sequence of Poincaré inequalities. It is worth noting that these inequalities consist essentially in a comparison between metrics on $X$. Namely, we compare the original metric on $X$ with all the pull-back metrics obtained from maps to metric spaces of $\mathcal{M}$. let us be more precise.

Let $X$ be a set. A pseudo-metric on $X$ is a function: $\sigma: X^{2} \rightarrow \mathbf{R}_{+}$such that $\sigma(x, y)=\sigma(y, x), \sigma(x, y) \leq \sigma(x, z)+\sigma(z, y)$, and $\sigma(x, x)=0$, for all $x, y, z \in X$. In the sequel, a pseudo-metric will simply be called a metric. 
If $(Y, d)$ is a metric space and $F: X \rightarrow Y$ is a map, then we can consider the pull-back metric $\sigma_{F}(x, y)=d(F(x), F(y))$, for all $x, y \in X$. Such metrics are called $Y$-metrics on $X$. More generally, if $\mathcal{M}$ is a class of metric spaces, a $\mathcal{M}$-metric on $X$ is a $Y$-metric for some $Y \in \mathcal{M}$.

Assume here that $X=(X, d)$ is a metric space. A metric $\sigma$ is a called coarse if there exist two increasing unbounded functions $\rho_{-}, \rho_{+}$such that, for all $x, y \in X$,

$$
\rho_{-}(d(x, y)) \leq \sigma(x, y) \leq \rho_{+}(d(x, y)) .
$$

Note that if $\sigma=\sigma_{F}$ is a $Y$-metric associated to a map $F: X \rightarrow Y$, then $\sigma_{F}$ is coarse if and only if $F$ is a coarse embedding.

Definition 9. A sheaf of metrics on a set $X$ is a collection of pairs $(\sigma, \Omega)$, where $\Omega$ is a subset of $X$, and $\sigma$ is a metric defined on $\Omega$. If $\Omega$ is an subset of $X$, we denote by $\mathcal{F}(\Omega)$ the set of pairs $(\sigma, \Omega) \in \mathcal{F}$.

We also assume that the restriction is well-defined from $\mathcal{F}(\Omega)$ to $\mathcal{F}\left(\Omega^{\prime}\right)$ for every $\Omega^{\prime} \subset \Omega$ (which is automatic in the case of sheaves of $\mathcal{M}$-metrics).

One checks easily that squares of Hilbert metrics, and more generally $p$-powers of $L^{p}$-metrics form a convex sub-cone of the space of real-valued functions on $X^{2}$. This is in fact a crucial remark for what follows.

Definition 10. Let $X$ be a set. A sheaf $\mathcal{F}$ of metrics on $X$ is called $p$-admissible (for some $p>0$ ) if for every $\Omega$, the following hold.

(i) The set of $\sigma^{p}$, where $\sigma \in \mathcal{F}(\Omega)$ forms a sub-cone of the space of functions on $\Omega^{2}$.

(ii) $\mathcal{F}(\Omega)$ is closed for the topology of pointwise convergence.

(iii) Let $\left(U_{i}\right)$ be a family of finite subsets whose union is $\Omega$, satisfying that for all $i, j \in I$, there exists $k$ such that $U_{i} \cup U_{j} \subset U_{k}$. Let $\left(\sigma_{i}, U_{i}\right)$ be a compatible family of sections, in the sense that $\sigma_{i}$ and $\sigma_{j}$ coincide on the intersection $U_{i} \cap U_{j}$. Then there exists a section $\sigma \in \mathcal{F}(\Omega)$, whose restriction to every $U_{i}$ is $\sigma_{i}$. In other words, $\mathcal{F}(\Omega)$ is the direct limit of the $\mathcal{F}\left(U_{i}\right)$.

Proposition 11. Let $X$ be a set, and let $\mathcal{M}$ be a class of metric spaces which is closed under ultra-limits. Then the sheaf of $\mathcal{M}$-metrics on (subsets of) $X$ satisfies conditions (ii) and (iii) of Definition 10.

Proof: That $X$ satisfies (ii) is trivial. Let us prove (iii). Fix a point $o \in \Omega$ and consider $\mathcal{A}$ the partially ordered (for the inclusion) set of all finite subsets of $\Omega$ containing $o$. For every $\sigma_{U} \in \mathcal{F}(U)$, where $U \in \mathcal{A}$, choose $Y_{F} \in \mathcal{M}$ and 
$F_{U}: U \rightarrow Y_{U}$ be such that $\sigma_{U}(x, y)=d\left(F_{U}(x), F_{U}(y)\right)$ for all $x, y \in U$. In every $Y_{U}$, take $y_{U}=F_{U}(o)$ for the origin. Fix an ultra-filter $\mathcal{U}$ on $\mathcal{A}$. Now, the limit $F$ of the $F_{U}$ is well defined from

$$
\Omega=\bigcup_{U \in A_{o}} U \rightarrow \lim _{\mathcal{U}}\left(Y_{U}, y_{U}\right)
$$

and $\sigma(x, y)=d(F(x), F(y))$ satisfies the third condition of Definition 10.

As a consequence of the proposition, we get the following examples.

Examples 12.

- for $p \geq 1$, the sheaf of $L^{p}$-metrics is $p$-admissible [H].

- Let $c>0$ and $1<p<\infty$. The class $\mathcal{M}_{c, p}$ of $(c, p)$-uniformly convex Banach spaces, is the class of uniformly convex Banach spaces whose moduli of convexity satisfy $\delta(t) \geq c t^{p}$. The sheaf of $\mathcal{M}_{c, p}$-metrics is $p$-admissible.

- The sheaf of CAT(0)-metrics is 2-admissible.

If $(X, \mu)$ is a probability space and $f$ is a integrable function on $X$, we denote $\mathbf{E}_{\mu}(f)=\int f(x) d \mu(x)$.

Theorem 13. Let $X$ be a metric space. Let $\mathcal{F}$ be a p-admissible sheaf of metrics on $X$. Then there exists no coarse metric in $\mathcal{F}(X)$ if and only if for every function $\rho_{+}: \mathbf{R}_{+} \rightarrow \mathbf{R}_{+}$, there exist $K>0$, and a sequence of symmetric probability measures $\left(\mu_{n}\right)$ with the following properties

- for every $n \in \mathbf{N}, \mu_{n}$ is supported on a finite subset $A_{n}$ of $\Delta_{n}(X)$;

- for every $n \in \mathbf{N}$ and every $\sigma \in \mathcal{F}\left(A_{n}\right)$ satisfying

$$
\sigma(x, y) \leq \rho_{+}(d(x, y)), \quad \forall(x, y) \in A_{n}
$$

one has

$$
\mathbf{E}_{\mu_{n}}\left(\sigma^{p}\right) \leq K^{p}
$$

Remark 14. Note that this theorem characterizes metric spaces that do not coarsely embed into $L^{p}$-spaces, $C A T(0)$-spaces, uniformly convex spaces... Indeed, by a theorem of Pisier [Pi], any uniformly convex Banach space is isomorphic to a $(c, p)$-uniformly convex Banach space for some $1<p<\infty$ and $c>0$. We can also avoid to use this deep theorem by defining $\phi$-admissible sheafs of metrics for any non-decreasing convex function $\phi$, and by adapting the proof of Theorem 13 to this slightly more general setting. 
Generalizing the case of Hilbert spaces, the previous theorem can be reformulated in terms of generalized expanders. Let $\mathcal{M}$ be a class of metric spaces.

Definition 15. A sequence of $(\mathcal{M}, p)$-valued generalized expanders is a sequence of finite metric spaces $\left(X_{n}\right)$ satisfying the following property. For every function $\rho_{+}: \mathbf{R}_{+} \rightarrow \mathbf{R}_{+}$, there exist $K>0$, and a sequence $r_{n} \rightarrow \infty$ such that each $X_{n}$ carries a symmetric probability measure $\mu_{n}$ satisfying

- $\mu_{n}$ is supported on $\Delta_{n}\left(X_{n}\right)$;

- for all maps $F$ from $X_{n}$ to a metric space $Y \in \mathcal{M}$, satisfying

$$
|F(x)-F(y)| \leq \rho_{+}(d(x, y)) \quad \forall(x, y) \in \Delta_{1}\left(X_{n}\right),
$$

we have

$$
\sum_{x, y \in X_{n}}|F(x)-F(y)|^{p} \mu_{n}(x, y) \leq K^{p}
$$

Corollary 16. Let $\mathcal{M}$ be a class of metric spaces such that the corresponding sheafs are $p$-admissible for some $1 \leq p<\infty$. Then a metric space $X$ does not coarsely embed into any element of $\mathcal{M}$ if and only if it has a coarsely embedded sequence of $(\mathcal{M}, p)$-valued generalized expanders.

\subsection{The invariant setting}

If $G$ is a countable discrete group, a length function on $G$ is a function $L$ : $G \rightarrow \mathbf{R}_{+}$satisfying $L(1)=0, L(g h) \leq L(g)+L(h)$, and $L\left(g^{-1}\right)=L(g)$ for all $g, h \in G$. Clearly, a length function on $G$ gives rise to a left-invariant metric $\sigma_{L}(g, h)=L\left(g^{-1} h\right)$. Conversely, given a left-invariant metric $\sigma$, we define a length function by $L(g)=\sigma(1, g)$.

We can define sheaves of length functions as we defined sheaves of metrics.

Definition 17. A sheaf of length functions $\mathcal{F}$ on a group $G$ is a family of pairs $(\Omega, L)$, where $\Omega$ is a symmetric neighborhood of 1 , and $L: \Omega \rightarrow \mathbf{R}_{+}$satisfying $L(1)=0, L(g h) \leq L(g)+L(h), L\left(g^{-1}\right)=L(g)$ for all $g, h \in \Omega$ such that $g h \in \Omega$.

Note that a sheaf of lengths naturally induces a sheaf of "locally invariant" metrics on $G$ by the relation $\sigma(g, h)=L\left(g^{-1} h\right)$ (whenever this is well defined). We will say that a sheaf of lengths is $p$-admissible if so is the corresponding sheaf of metrics.

Example 18. If $\mathcal{M}$ is a class of metric spaces, the sheaf of $\mathcal{M}$-lengths on $G$ is the set of $(\Omega, L)$ as above, where $L(g)=\sigma(1, g)$ for some $\mathcal{M}$-metric $\sigma$ defined on $\Omega^{2}$, satisfying $\sigma\left(h g, h g^{\prime}\right)=\sigma\left(g, g^{\prime}\right)$ for all $g, g^{\prime}, h \in G$ such that $g, g^{\prime}, h g, h g^{\prime} \in \Omega$. 
Let $G$ be a group equipped with a length function $L_{0}$. Then, one sees immediately that the proof of Theorem 13 can be formulated with length functions instead of metrics, which yields the following result

Theorem 19. Let $\mathcal{F}$ be a p-admissible sheaf of length functions on $G$. Then there exists no coarse length in $\mathcal{F}(G)$ if and only if there exist $K>0$, and for every $n \in \mathbf{N}$, a symmetric, finitely supported probability measure $\mu_{n}$ on $\left\{g, L_{0}(g) \geq n\right\}$ with the following property: for every $L \in \mathcal{F}(G)$ satisfying

$$
L(g) \leq L_{0}(g), \quad \forall g \in G,
$$

one has

$$
\mathbf{E}_{\mu_{n}}\left(L^{p}\right) \leq K^{p}
$$

\subsection{Quantitative statements}

Let $\mathcal{F}$ be a sheaf of metrics on $X$. The $\mathcal{F}$-compression rate of $X$, denoted by $R_{\mathcal{F}}(X)$ is the supremum of all $\alpha>0$ such that there exists $\sigma \in \mathcal{F}(X)$ satisfying $d(x, y)^{\alpha} \leq \sigma(x, y) \leq d(x, y)$, for $d(x, y)$ large enough. The Hilbert compression rate, usually denoted by $R(X)$, has been introduced in [GK] and studied by many authors since then as it provides an interesting quasi-isometry invariant of finitely generated groups.

A slight modification of the proof of Theorem 13 yields

Theorem 20. Let $X$ be a metric space. Let $\mathcal{F}$ be a p-admissible sheaf of metrics on $X$. The $\mathcal{F}$-compression rate of $X$ is at most $\alpha$ if and only if for all $\beta>\alpha$, there exist $K>0$, and for every $n \in N$, a symmetric, finitely supported probability measure $\mu_{n}$ on $\Delta_{n}(X)$, with the following property: for every $\sigma \in \mathcal{F}(X)$ satisfying

$$
\sigma(x, y) \leq d(x, y), \quad \forall(x, y) \in \Delta_{1}(X)
$$

one has

$$
\mathbf{E}_{\mu_{n}}\left(\sigma^{p}\right) \leq\left(K n^{\beta}\right)^{p} .
$$

Assume that $X=G$ is a finitely generated group equipped with a word metric, denoted by $|g|=|g|_{S}$, associated to a finite symmetric generating subset $S$. Theorem 20 becomes

Theorem 21. Let $\mathcal{F}$ be a p-admissible sheaf of length functions on $G$. The $\mathcal{F}$ compression rate of $G$ is at most $\alpha$ if and only if there exist $K>0$, and for every $n \in \mathbf{N}$, a symmetric, finitely supported probability measure $\mu_{n}$ on $\{g,|g| \geq n\}$ with the following property: for every $L \in \mathcal{F}(G)$ satisfying

$$
L(g) \leq|g|, \quad \forall g \in G
$$


one has

$$
\mathbf{E}_{\mu_{n}}\left(L^{p}\right) \leq\left(K|g|^{\beta}\right)^{p}
$$

\section{Proof of Theorem 13}

The "if" part is obvious, as the condition (3.1) roughly says that the sequence $\mu_{n}$ selects pairs $(x, y)$ of arbitrarily distant points in $X$ for which $\sigma(x, y) \leq K$.

Let $X$ be a metric space, and let $\mathcal{F}$ be a $p$-admissible sheaf of metrics on $X$. We assume that $\mathcal{F}(X)$ contains no coarse metric. Let $\rho_{+}: \mathbf{R}_{+} \rightarrow \mathbf{R}_{+}$be an increasing unbounded function.

Our first step is the next lemma. Let $\mathcal{A}$ be the set of finite subsets of $X$ containing a distinguished point $o$.

Lemma 22. Assume that there exists a function $T: \mathbf{R}_{+} \rightarrow \mathbf{R}_{+}$with the following property: for all $U \in \mathcal{A}$, and all $K>0$, there exists $\sigma \in \mathcal{F}(U)$ such that

$$
\sigma(x, y) \leq \rho_{+}(d(x, y)), \quad \forall x, y \in U
$$

and

$$
\sigma(x, y) \geq T(K)
$$

for all $(x, y) \in \Delta_{T}(U)$. Then there exists a coarse element in $\mathcal{F}(X)$.

Proof: As $\mathcal{F}$ is $p$-admissible, up to taking a pointwise limit with respect to an ultrafilter on $\mathcal{A}$, we can assume that for all $K>0$, there exists $\sigma \in \mathcal{F}(X)$ such that

$$
\sigma(x, y) \leq \rho_{+}(d(x, y)), \quad \forall x, y \in X
$$

and

$$
\sigma(x, y) \geq K
$$

for all $(x, y) \in \Delta_{T(K)}(X)$.

Let $K_{n}$ be an increasing sequence satisfying

$$
\sum_{n=1}^{\infty} \frac{1}{K_{n}^{p}} \leq 1
$$

and let $T$ be as in the lemma. Now take $\sigma_{n}$ as above, and define

$$
\sigma(x, y)=\left(\sum_{n \geq 1}\left(\sigma_{n}(x, y) / K_{n}\right)^{p}\right)^{1 / p}
$$


for all $x, y \in X$. The fact that $\sigma$ is well defined follows from the fact that $\mathcal{F}$ is $p$-admissible. Moreover, we have

$$
\rho_{-}(d(x, y)) \leq \sigma(x, y) \leq \rho_{+}(d(x, y))
$$

for all $x, y \in X$, where

$$
\rho_{-}(t)=\operatorname{card}\left\{n, T\left(K_{n}\right) \leq t\right\} .
$$

Clearly, $\rho_{-}(t) \rightarrow \infty$ when $t \rightarrow \infty$, so we are done.

The second step of the proof is an adaptation of the proof of [M1, Proposition 15.5.2]. Suppose that $X$ does not coarsely embed into a Hilbert space.

By the lemma, there exists a number $K$ with the following property. For all $T$, there exist $U \in A$ such that for all $\sigma \in \mathcal{F}(U)$ satisfying

$$
\sigma(x, y) \leq \rho_{+}(d(x, y))
$$

there are two points $x, y$ in $U$ such that $T \leq d(x, y)$ and

$$
\sigma(d(x, y))<K
$$

Note that we can take $T$ such that $\rho_{+}(T) \geq K$.

Consider the two following convex subsets of $\ell^{2}\left(U^{2}\right)$. Let $C_{1}$ be the set of functions $\phi: U^{2} \rightarrow \mathbf{R}_{+}$satisfying

$$
\phi(x, y) \leq \rho_{+}(d(x, y))^{p}, \quad \forall(x, y) \in U^{2},
$$

and

$$
\phi(x, y) \geq K^{p}, \quad \forall(x, y) \in \Delta_{T}(U) .
$$

Let $C_{2}$ be the set of $\sigma^{p}$, where $\sigma \in \mathcal{F}(U)$ satisfies

$$
\sigma(x, y) \leq \rho_{+}(d(x, y))
$$

The previous reformulation of the lemma implies that these two convex subsets are disjoint. We have even better. For every subset $V$ of a vector space $E$, we denote

$$
\mathbf{R}_{+} V=\left\{t v, t \in \mathbf{R}_{+}, v \in V\right\} .
$$

Lemma 23. The cones $\mathbf{R}_{+} C_{1}$ and $\mathbf{R}_{+} C_{2}$ intersect only at $\{0\}$. Moreover, $\{0\}$ is extremal in both cones. 
Proof: The fact that $\{0\}$ is extremal is just a consequence of the fact that the two cones only contain non-negative functions. Let $t>0$ and let $\phi \in C_{2} \backslash\{0\}$. We want to prove that $t \phi$ does not belong to $C_{1}$. By the first condition of $p$ admissibility, there exists $\sigma_{t} \in \mathcal{F}(U)$ such that $t \phi=\sigma_{t}^{p}$. Moreover, if $t \phi$ also satisfies (4.2), then $\sigma_{t}$ satisfies (4.1), so $t \phi \in C_{2}$, and hence it cannot be in $C_{1}$.

Hence by Hahn-Banach's theorem, there exists a vector $u \in \ell^{2}\left(U^{2}\right)$ such that

$$
\langle\phi, u\rangle>0
$$

for all non-zero $\phi \in C_{1}$ and

$$
\langle\phi, u\rangle<0
$$

for all non-zero $\phi \in C_{2}$.

Let $u_{+}=\max \{u, 0\}$ and $u_{-}=\min \{u, 0\}$. One sees that $u_{+}$is non-zero in restriction to $\Delta_{T}(U)$ by applying the first inequality to the function

$$
\phi(x, y)= \begin{cases}0, & \text { if } d(x, y)<T \\ K^{p}, & \text { otherwise }\end{cases}
$$

Now, apply the first inequality to the function

$$
\phi(x, y)= \begin{cases}K^{p}, & \text { if } u(x, y)>0, \text { and } d(x, y) \geq T \\ 0, & \text { if } u(x, y)>0, \text { and } d(x, y)<T \\ \rho_{+}(d(x, y))^{p}, & \text { otherwise }\end{cases}
$$

Note that this is possible as $T$ has been chosen such that $\rho_{+}(T) \geq K$. We get

$$
\sum_{x, y} \rho_{+}(d(x, y))^{p} u_{-}(x, y) \leq K^{p} \sum_{(x, y) \in \Delta_{T}(U)} u_{+}(x, y) .
$$

On the other hand, if $\phi \in C_{2}$, i.e. $\phi(x, y)=\sigma(x, y)^{p}$, then using the second inequality,

$$
\begin{aligned}
\sum_{(x, y) \in \Delta_{T}(B(o, T))} \sigma(x, y)^{p} u_{+}(x, y) & \leq \sum_{(x, y) \in B(o, T)^{2}} \sigma(x, y)^{p} u_{+}(x, y) \\
& \leq \sum_{x, y} \sigma(x, y)^{p} u_{-}(x, y) \\
& \leq \sum_{x, y} \rho_{+}(d(x, y))^{p} u_{-}(x, y) .
\end{aligned}
$$

Now, combining these two inequalities, we get

$$
\sum_{x, y} \sigma(x, y)^{p} \frac{u_{+}(x, y)}{\sum_{(x, y) \in \Delta_{T}(U)} u_{+}(x, y)} \leq K^{p} .
$$


So the theorem follows by taking the probability measure on $\Delta_{T}(U)$ defined by

$$
\mu(x, y)=\frac{u_{+}(x, y)}{\sum_{(x, y) \in \Delta_{T}(U)} u_{+}(x, y)} .
$$

\section{References}

[A] P. Assouad. Plongements lipschitziens dans $\mathbf{R}^{n}$. Bull. S.M.F. 111, 429-448, 1983.

[AW] C. A. Akemann, M. E. Walter. Unbounded negative definite functions. Canad. J. Math. 33 4, 862-871, 1981.

[Sch] I. J. SchoenberG. Metric spaces and completely monotone functions. Annals of Math. 39(4), 811-841, 1938.

[C1] Y. Cornulier. Relative Kazhdan Property. Ann. Sci. Ecole Norm. Sup. 39(2), 301-333, 2006

[C2] Y. CoRnulier. Kazhdan and Haagerup Properties in algebraic groups over local fields. J. Lie Theory 16, 67-82, 2006.

[CTV] Y. Cornulier, R. TesserA, Alain VAlette. Isometric group actions on Hilbert spaces: growth of cocycles. Geom. Funct. Anal. 17 (2007), 770-792.

[H] S. HeInRICH Ultraproducts in Banach space theory. J. Reine Angew. Math. 313, 72-104, 1980.

[GK] E. Guentner, J. Kaminker. Exactness and uniform embeddability of discrete groups. J. London Math. Soc. 70, 703-718, 2004.

[JR] W. B. Johnson and N. L. RANDRIANARIVONY. $\ell_{p}(p>2)$ does not coarsely embed into a Hilbert space. Manuscript, 2004.

[JS] M. JeRrum and A. SinclaIR, Conductance and the rapid mixing property for Markov chains: The approximation of the permanent resolved; Proceedings of the 20th ACM Symposium on Theory of Computing, 235-244, 1988.

[L] A. Lubotzky, Discrete groups, expanding graphs and invariant measures, Progress in Mathematics, Vol. 125, Birkhauser Verlag, 1994.

[M1] J. Matousek. Lecture on Discrete Geometry. Springer-Verlag, New York. 2002. 
[M2] J. MatouseK. On embedding expanders into $\ell_{p}$ spaces. Israel J. of Math. 102, 189-197, 1996.

[Pi] G. Pisier. Martingales with values in uniformly convex spaces. Israel J. Math. 20, 326-350, 1975.

[T] R. Tessera. Quantitative property A, Poincaré inequalities, $L^{p}$-compression. and $L^{p}$-distortion for metric measure spaces. math.MG/0702384.

[MN] A. NAOR and M. Mendel. Metric cotype. to appear in the Ann. of Math.

Romain Tessera

Department of mathematics, Vanderbilt University,

Stevenson Center, Nashville, TN 37240.

E-mail: romain.a.tessera@vanderbilt.edu 